\title{
On-demand Versus Continuous Maintenance Treatment of Gastroesophageal Reflux Disease With Proton Pump Inhibitors: A Systematic Review and Meta-analysis
}

\author{
Seung Joo Kang, ${ }^{1}$ Hye-Kyung Jung, ${ }^{2}$ Chung Hyun Tae, ${ }^{2}$ Seung Young Kim, ${ }^{3}$ and Kwang Jae Lee ${ }^{4 *}$ \\ ${ }^{\text {I} D e p a r t m e n t ~ o f ~ I n t e r n a l ~ M e d i c i n e, ~ S e o u l ~ N a t i o n a l ~ U n i v e r s i t y ~ H o s p i t a l ~ G a n g n a m ~ C e n t e r, ~ S e o u l, ~ K o r e a ; ~}{ }^{2}$ Department of Internal Medicine, Ewha \\ Womans University College of Medicine, Seoul, Korea; ${ }^{3}$ Department of Internal Medicine, College of Medicine, Korea University, Seoul, Korea; \\ and ${ }^{4}$ Department of Gastroenterology, Ajou University School of Medicine, Suwon, Gyeonggi-do, Korea
}

\section{Background/Aims}

Long-term maintenance treatment of gastroesophageal reflux disease (GERD) is commonly used to prevent relapse of reflux symptoms; however, due to concerns about safety of long-term proton pump inhibitors (PPI) use, on-demand therapy is recommended as a longterm treatment modality. We compared the efficacy of on-demand and continuous PPI therapy for maintenance treatment of patients with GERD using meta-analysis.

\section{Methods}

Core electronic databases were searched for randomized controlled trials comparing on-demand and continuous therapy in GERD patients. The primary outcome was treatment failure of maintenance therapy, and the secondary outcomes included symptomatic relief, patient satisfaction, and amount of PPI use.

\section{Results}

Overall, 11 studies were selected in the systematic review and meta-analysis. Compared with continuous PPI therapy, on-demand therapy showed similar outcomes for treatment failure (risk ratio, 1.26; 95\% confidence interval [Cl], 0.76-2.07), particularly in the non-erosive esophageal reflux disease and mild erosive reflux disease group (risk ratio, 1.48; $95 \% \mathrm{Cl}, 0.39-5.63$ ). In studies including severe esophagitis patients, continuous PPI maintenance treatment was more effective $(\beta, 0.127[95 \% \mathrm{Cl}, 0.066-0.188] ; P<0.001)$. Severity of esophagitis was associated with higher efficacies of continuous maintenance therapy. The amount of daily PPI use was about half in the on-demand group compared to the continuous group (risk difference $-0.52 ; 95 \% \mathrm{Cl},-0.62--0.42$ ).

\section{Conclusions}

On-demand PPI therapy shows comparable efficacy to the continuous maintenance treatment in the non-erosive esophageal reflux disease and mild erosive reflux disease group, and can remarkably reduce the amount of PPI use. Therefore, on-demand therapy may be preferentially recommended in the maintenance treatment of GERD unaccompanied by severe esophagitis.

(J Neurogastroenterol Motil 2022;28:5-14)

Key Words

Gastroesophageal reflux; Maintenance; Proton pump inhibitors

Received: May 14, 2021 Revised: July 30, 2021 Accepted: August 10, 2021

() This is an Open Access article distributed under the terms of the Creative Commons Attribution Non-Commercial License (http://creativecommons. org/licenses/by-nc/4.0) which permits unrestricted non-commercial use, distribution, and reproduction in any medium, provided the original work is properly cited.

${ }^{*}$ Correspondence: Kwang Jae Lee, MD, PhD Department of Gastroenterology, Ajou University School of Medicine, 164 World Cup-ro, Yeongtong-gu, Suwon, Gyeonggi-do 16499, Korea

Tel: +82-31-219-5119, Fax: +82-31-219-5999, E-mail: kjl@ajou.ac.kr 


\section{Introduction}

Gastroesophageal reflux disease (GERD) is a chronic digestive disorder resulting from the reflux of gastric contents into the esophagus that is often accompanied by troublesome symptoms of heartburn, acid regurgitation, or other extra-esophageal symptoms such as chest pain, chronic cough, hoarseness or globus. ${ }^{1}$ GERD imposes an important burden of illness worldwide with an estimated worldwide prevalence of between $8 \%$ and $33 \% .{ }^{2}$ GERD is heterogeneous and presented with different phenotypes. Erosive reflux disease (ERD) is defined as mucosal defect by endoscopy and occurs in approximately $25 \%$ of patients with GERD symptoms. ${ }^{3}$ Non-erosive reflux disease (NERD) is present in approximately $70 \%$ of patients and is characterized by the presence of typical GERD symptoms associated with pathological acid reflux but the absence of demonstrable esophageal mucosal injury on endoscopy. ${ }^{4}$

Currently, acid suppressive therapy with proton pump inhibitors (PPIs) has proved to be the most effective treatment strategy for GERD patients and is recommended as a first-line treatment. ${ }^{5}$ PPIs have shown superiority over histamine H2-receptor antagonists for controlling symptoms as well as for healing erosions. ${ }^{6} \mathrm{Al}-$ though symptomatic relief and acute healing of esophageal lesions can be achieved by short-term PPI treatments, up to $75 \%$ of patients with NERD and up to $90 \%$ of patients with ERD experience relapse within 6 months to 1 year after termination of initial treatments. ${ }^{7}$ Therefore, long-term continuous maintenance treatment with PPIs is required for the majority of patients with GERD to adequately control symptoms and to heal mucosal lesions. However, recent studies suggest that long-term PPI treatment can increase the risk of Clostridium difficile or other enteric infection, kidney disease, bone fractures, or micronutrient deficiency. ${ }^{8}$ In addition to safety issues, continuous maintenance may have led to unnecessary use of PPIs, increasing overall costs. In the United States, the total expenditure for PPI treatment was over $\$ 11$ billion annually. Therefore, maintenance therapy such as "on-demand" PPI therapy or "intermittent" PPI therapy have been used in clinical practice and their effectiveness has also been studied. On-demand PPI therapy means that patients take a daily dose of a PPI when symptoms recur and stop medication when symptoms resolve. Intermittent PPI therapy is when patients take a regular daily dose of a PPI upon symptom relapse and continue for a pre-specified duration, which is typically 1 or 2 weeks regardless of symptom response. To date, there have been 2 meta-analysis studies comparing on-demand and continuous PPI therapy. Boghossian et $\mathrm{al}^{10}$ performed a meta-analysis to compare the effects of continuous PPI therapy with stopping or on-demand PPI therapies in $2017 . .^{10}$ The authors concluded that on-demand PPI therapy may increase risk of "lack of symptom control" compared with continuous PPI use (risk ratio $[\mathrm{RR}], 1.71 ; 95 \%$ confidence interval $[\mathrm{CI}], 1.31-2.21$ ), which means lower efficacy of on-demand therapy. However, Khan et $\mathrm{al}^{11}$ reported that on-demand PPI therapy was superior to continuous PPI use in terms of treatment failure (RR, 1.71; 95\% CI, 1.31-2.21) in patients with NERD and mild erosive esophagitis. ${ }^{11}$ These 2 meta-analysis results have drawn conflicting conclusions. Therefore, in this meta-analysis, we intended to comprehensively analyze the treatment failure rates, patient satisfaction, the amount of PPI usage, and symptom relief effects between on-demand PPI and continuous maintenance by synthesizing the studies for GERD patients. In addition, we preformed meta-regression to determine whether there is a difference in the effectiveness of 2 treatments according to the presence and severity of ERD and ethnicities.

\section{Materials and Methods}

This meta-analysis was conducted in accordance with the principles of the Preferred Reporting Items for Systematic Reviews and Meta-Analysis (PRISMA) statement. ${ }^{12}$ The need for approval from the institutional review board was waived since this study was performed by reviewing the literatures.

\section{Literature Search Strategy}

We searched all relevant studies that evaluated with efficacy of on-demand PPI maintenance and continuous PPI maintenance in GERD patients using PubMed, EMBASE, the Cochrane library, and KoreaMed from inception to December 2020. The following search terms were used: ([gastroesophageal reflux] or [reflux esophagitis] or [erosive esophagitis] or [heartburn]) and ([proton pump inhibitors] or [PPIs]) and ([maintenance] or [on-demand] or [continuous]). The detailed search strategies in each database and the search results are shown in Supplementary Table 1. Cited references in published studies were manually searched to identify other relevant studies.

\section{Study Selection}

The inclusion criteria in this study were as follows: (1) patients with GERD including uninvestigated GERD, NERD, and ERD who need PPI maintenance therapy; (2) comparison of on-demand PPI maintenance treatment with continuous PPI maintenance treatment; (3) randomized controlled trials (RCTs) with parallel 
design; (4) adults aged over 18 years; and (5) studies available in full-text form. The exclusion criteria were as follows: (1) abstractonly publications or unpublished studies, (2) case reports and narrative reviews, (3) systematic review/meta-analysis, (4) proceedings or study protocols, and (5) studies with insufficient data regarding treatment outcomes. In the first stage of study selection, irrelevant articles were excluded by reviewing the titles and abstracts of the studies retrieved by keyword search. Thereafter, the full texts of selected studies were reviewed in accordance with our inclusion and exclusion criteria.

\section{Quality Assessment}

All studies were assessed using Cochrane's "Risk of Bias" tool, which includes the following domains: random sequence generation (selection bias), allocation concealment (selection bias), blinding of participants and personnel (performance bias), blinding of outcome assessment (detection bias), incomplete outcome data addressed over the short and long terms (attrition bias), selective reporting (reporting bias), and other biases. ${ }^{13}$ Two authors (S.J.K. and C.H.T.) independently assessed the methodological quality of all included studies, and any disagreements between the 2 evaluators were resolved by discussion and consensus. If no agreement could be reached, a third investigator (H.K.J.) was consulted.

\section{Data Extraction}

The primary outcome of this meta-analysis was treatment failure with maintenance therapy, which means that patients discontinued the allocated maintenance therapy prematurely. The secondary endpoints were as follows: (1) proportion of patients who achieved successful symptom relief, (2) satisfaction with maintenance therapy, (3) amount of pill usage in each maintenance therapy, and (4) adverse events in both treatment groups. Using a data extraction form that had been developed in advance, 2 reviewers (S.J.K. and C.H.T.) independently extracted the following information: first author, year of publication, study design, country, study period, PPI maintenance dosage, symptom evaluation tools, rates of treatment failure, degree of satisfaction, pill usage during maintenance, and adverse events.

\section{Statistical Methods}

Meta-analyses were performed to calculate the pooled RRs with a 95\% CI. The Mantel-Haenszel random-effects model was used for binary outcomes, and the inverse variance method was used for continuous outcomes. In addition, we evaluated subgroup analyses according to symptomatic GERD patients who did not undergo endoscopic evaluation, patients with NERD and patients with ERD. Heterogeneity was assessed using the $I^{2}$ test to calculate the percentage of total variation across the included articles. Negative values for $I^{2}$ were set to zero, and $I^{2} \geq 50 \%$ indicated the presence of substantial heterogeneity. Meta-regression was performed to investigate sources of heterogeneity (presence and severity of esophagitis and ethnicity). Publication bias was assessed qualitatively by inspecting funnel plots of the logarithmic RR vs their standard errors and quantitatively by Egger's linear regression method test and Begg's rank correlation test when 7 or more studies were available. All $P$-value were 2 -tailed, and $P<0.05$ was considered statistically

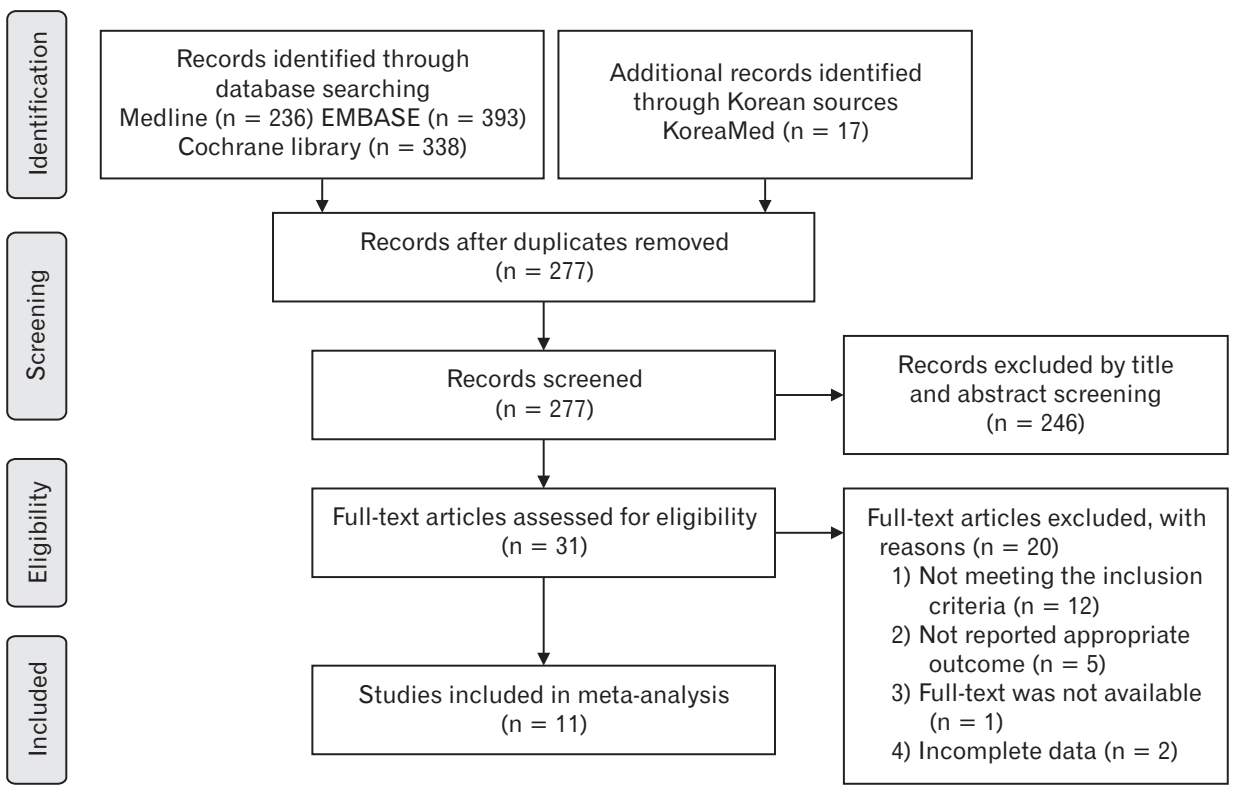

Figure 1. Flow diagram of the studies included in the meta-analysis. 
significant in all tests. For meta-analysis, we used Review Manager version 5.3 (RevMan for Windows, the Nordic Cochrane Center, Copenhagen, Denmark) and "meta" packages in R version 3.6.3.

\section{Results}

\section{Study Selection}

The flow diagram of the selection process, performed in accordance with PRISMA guidelines, is shown in Figure 1. The electronic database search identified a total of 630 records, of which 277 remained after removing duplicates. After screening the titles and abstracts, we excluded 246 irrelevant articles. The full texts of the 31 remaining articles were reviewed for eligibility. Of these, 11 studies met the inclusion criteria, and 20 studies were excluded from the final analysis.

\section{Characteristics of Included Studies}

The characteristics of the 11 included studies are summarized in Supplementary Table $1 .{ }^{14-24}$ The studies were RCTs published between 2004 and 2018, consisting of a total of 6138 GERD patients. The study design was a randomized, open-label study except for 1 study by van der Velden et $\mathrm{al}^{24}{ }^{24}$ which was a randomized, double-blind study. Two studies ${ }^{16,20}$ were conducted in Asian countries, and all other studies were performed in Western countries. Three studies by Hansen et al, ${ }^{17}$ Morgan et al, ${ }^{19}$ and Szucs et $\mathrm{al}^{22}$ included patients with GERD symptoms. Two studies by Bayerdorffer et $\mathrm{al}^{14}$ and Tsai et $\mathrm{al}^{23}$ included patients with NERD. The remaining 6 studies were performed in patients with ERD proven by endoscopy. The detailed GERD definition in each study is described in Supplementary Table 1. Except for 2 studies, ${ }^{16,23}$ the PPI doses for on-demand and continuous maintenance treatment groups were

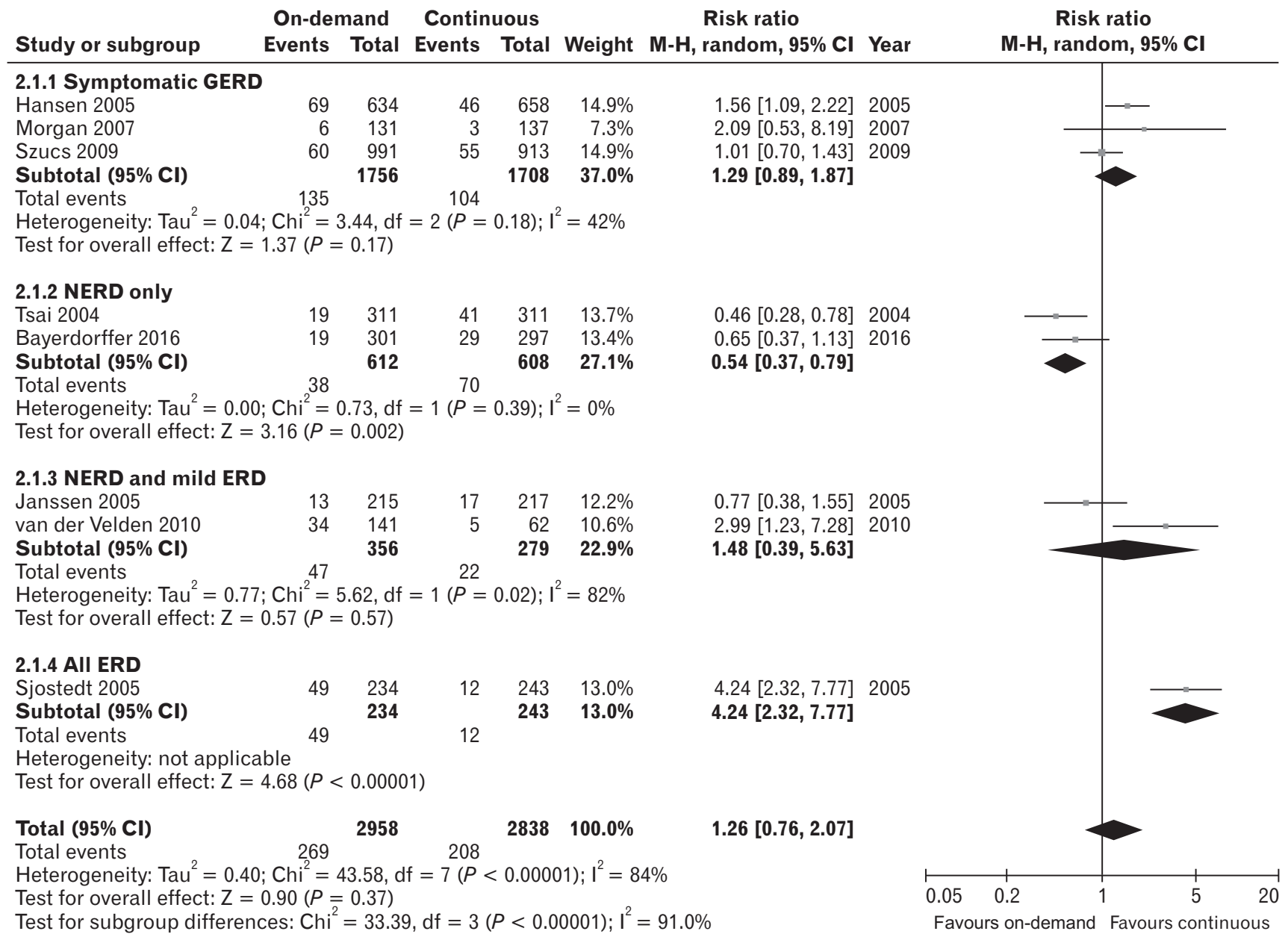

Figure 2. Forest plot of the relative efficacy in terms of the treatment failure between on-demand therapy and continuous therapy. M-H, MantelHaenszel; GERD, gastroesophageal reflux disease; NERD, non-erosive esophageal reflux disease; ERD, erosive reflux disease. 
the same. In the study by Cho et al, ${ }^{16} 40 \mathrm{mg}$ esomeprazole was used in the on-demand group and $20 \mathrm{mg}$ esomeprazole was used in the continuous group. In the study by Tsai et al, ${ }^{23} 20 \mathrm{mg}$ esomeprazole was used in the on-demand group and $15 \mathrm{mg}$ lansoprazole was used in the continuous group. The duration of maintenance therapy in the 2 studies by Cho et $\mathrm{al}^{16}$ and van der Velden et $\mathrm{al}^{24}$ was 12 and 13 weeks, respectively, and the remaining studies were maintained for 6 months. The risk of bias graph and risk of bias summary of included studies are presented in Supplementary Figure 1 and Supplementary Figure 2, respectively.

\section{Primary Outcomes}

A meta-analysis was performed on 8 RCTs that analyzed outcomes for treatment failure. The detailed outcome measurements of each study are summarized in Supplementary Table 2. Treatment failure was observed in 269 of 2958 patients (9.1\%) in the on-demand PPI group, and 208 of 2838 patients (7.3\%) in the continuous PPI group (Fig. 2). The RR with 95\% CI was 1.26 (0.76-2.07) $(P=0.372)$ which means no significant difference between 2 treatments in overall GERD patients. The heterogeneity of studies was $84 \%$. Funnel plot of 8 studies was shown in Supplementary Figure 3 and showed no significant asymmetry. No significant publication bias was detected from Egger's linear regression method test $(P$ $=0.674)$ and Begg's rank correlation test $(P=0.458)$. Subgroup analysis was performed according to the presence or absence of erosive esophagitis. We divided the entire patient group into the symptomatic GERD, NERD only, NERD and mild ERD, and all

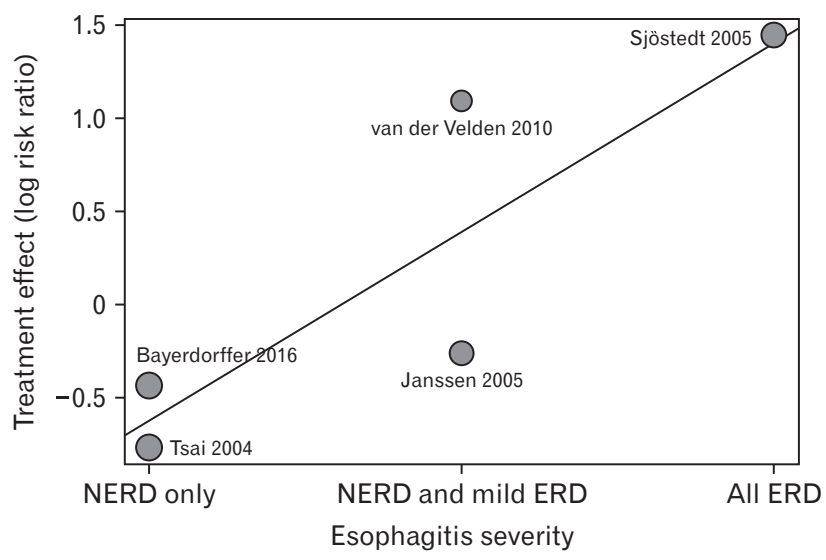

Fiǵure 3. Random-effects univariate meta-regression between treatment failure and esophagitis severity. Each circle represents a study and the size of the circle reflects the influence of that study on the model. The regression prediction is presented by the solid line (1.013 [95\% CI, 0.466-1.561], $P<0.001$ ). NERD, non-erosive esophageal reflux disease; ERD, erosive reflux disease.
ERD groups as shown in Figure 2. The mild ERD group includes patients with Los Angeles (LA) grade A or B or Savary-Miller grade 1 or 2. No significant difference in treatment failure between the on-demand and continuous groups was found upon metaanalysis in the symptomatic GERD group without endoscopy (RR, $1.29 ; 95 \% \mathrm{CI}, 0.89-1.87)$, and the NERD and mild ERD group (RR, 1.48; 95\% CI, 0.39-5.63). In the NERD only group, treatment failure was significantly lower in the on-demand group (RR, $0.54 ; 95 \% \mathrm{CI}, 0.37-0.79)$. However, in a study including patients with severe erosive esophagitis, treatment failure was significantly lower in the continuous PPI group (RR, 4.24; 95\% CI, 2.32-7.77). We performed meta-regression by presence and severity of esophagitis by endoscopy as shown in Figure 3. Severity of esophagitis was associated with higher efficacies of continuous maintenance therapy ( $\beta, 1.013$ [95\% CI, 0.466-1.561]; $P<0.001$ ) (Supplementary Table 2). Considering the high heterogeneity of analysis results, subgroup analysis was performed according to PPI types. There was no difference in treatment failure between continuous and ondemand maintenance treatments in the esomeprazole group and other PPI groups (Supplementary Fig. 4 and 5). We also carried out a sensitivity analysis to determine if the omission of each study would undermine the results of meta-analysis. The results of the changes were not obtained after omitting each study, indicating the stability of our analysis (Supplementary Table 3).

\section{Secondary Outcomes}

The proportion of patients who achieved successful symptom relief was higher in the continuous PPI group (RR, 1.16; 95\% CI, 1.05-1.29) (Fig. 4). Funnel plot of 9 studies is shown in Supplementary Figure 6. No significant publication bias was detected from Egger's linear regression method test $(P=0.469)$ and Begg's rank correlation test $(P=0.835)$. The heterogeneity among studies was significant $\left(I^{2}=91 \%\right)$. Therefore, we performed subgroup analysis according to ethnicity and the presence of esophagitis. There was no significant difference between therapies in the Western symptomatic GERD group (RR, 1.22; 95\% CI, 0.94-1.59), in Western NERD only group (RR, 1.05; 95\% CI, 0.98-1.13), and in Asian NERD and ERD groups (RR, 1.00; 95\% CI, 0.76-1.32). However, continuous PPI maintenance treatment showed more effectiveness in Western NERD and mild ERD group (RR, 1.17; 95\% CI, 1.071.28 ) and Western ERD group (RR, 1.37; 95\% CI, 1.23-1.52). When the study patient was limited to the NERD and the mild ERD group, continuous maintenance treatment showed a better effect (RR, 1.09; 95\% CI, 1.01-1.18) and the number needed to treat (NNT) was 14.3 (95\% CI, 7.7-20.9). To identify the sources 


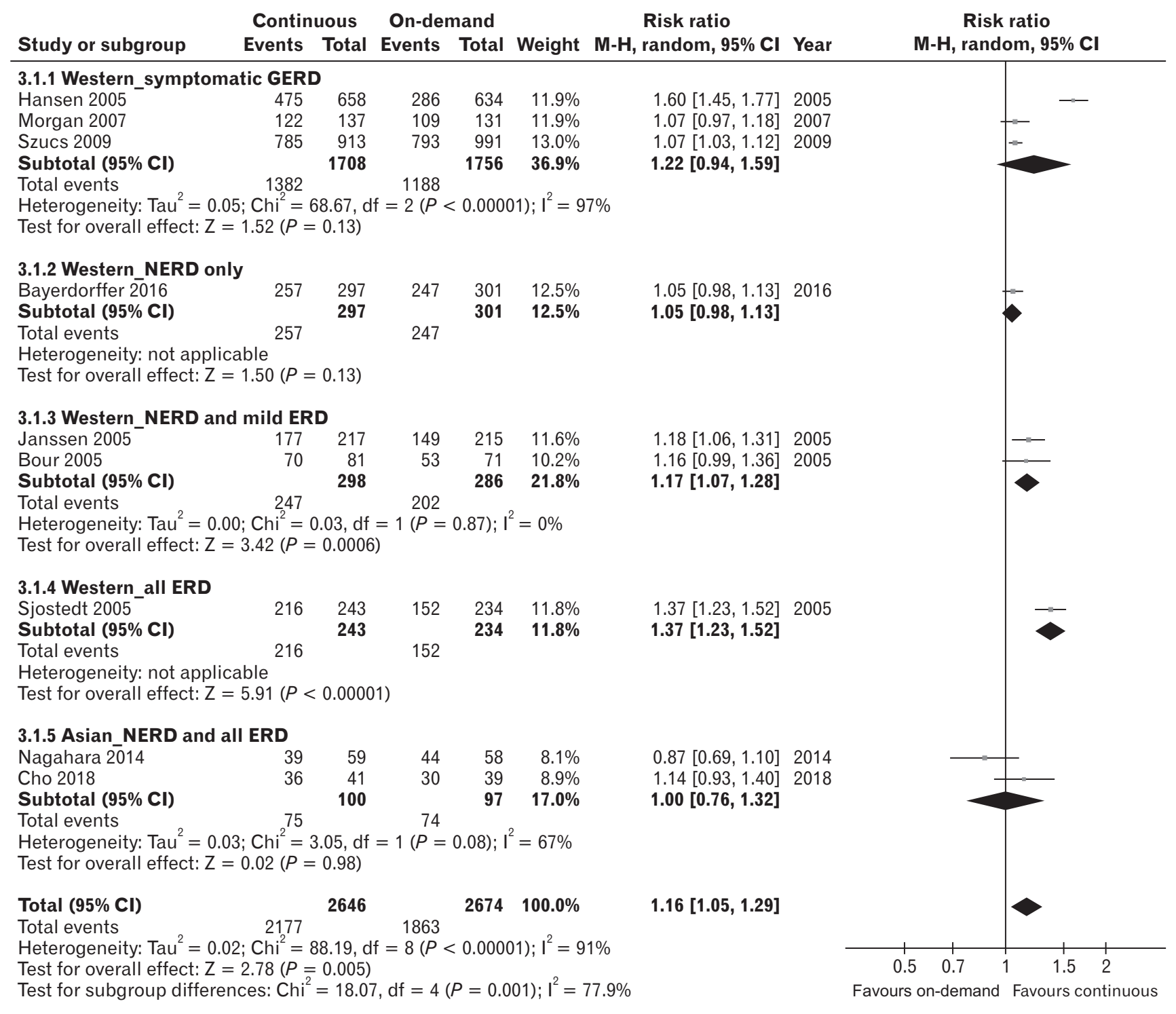

Figure 4. Forest plot of the relative efficacy in terms of the symptom relief between on-demand therapy and continuous therapy. M-H, MantelHaenszel; GERD, gastroesophageal reflux disease; NERD, non-erosive esophageal reflux disease; ERD, erosive reflux disease.

of heterogeneity, we performed meta-regression by the presence of esophagitis and ethnicity (Supplementary Fig. 7 and 8). In studies including severe esophagitis patients, continuous PPI maintenance treatment was more effective ( $\beta, 0.127$ [95\% CI, 0.066-0.188]; $P<0.001)$, but no significant difference was observed according to ethnicity $(\beta,-0.179[95 \% \mathrm{CI},-0.462-0.105] ; P=0.218)$ (Supplementary Table 2). We also performed subgroup analysis according to PPI types. Continuous therapy was more effective in the studies using esomeprazole, but there was no difference between the 2 groups in the studies using other PPI groups (Supplementary Fig. 9 and 10). Sensitivity analysis did not show changes after omit- ting each study, indicating the robustness of analysis as shown in Supplementary Table 3 .

Pill usage was significantly lower in the on-demand group as shown in Figure 5. In the on-demand group, pill usage per day was less than in the continuous group (risk difference, $-0.52 ; 95 \% \mathrm{CI}$, -0.62--0.42), which means that half of the PPI was used in the on-demand group compared to the continuous group. Patient satisfaction was measured in 6 RCTs. No significant difference in patient satisfaction was found between the 2 groups upon meta-analysis (RR, 0.97; 95\% CI, 0.93-1.01) (Supplementary Fig. 11). The frequency of adverse events did not differ between the 2 treatment 


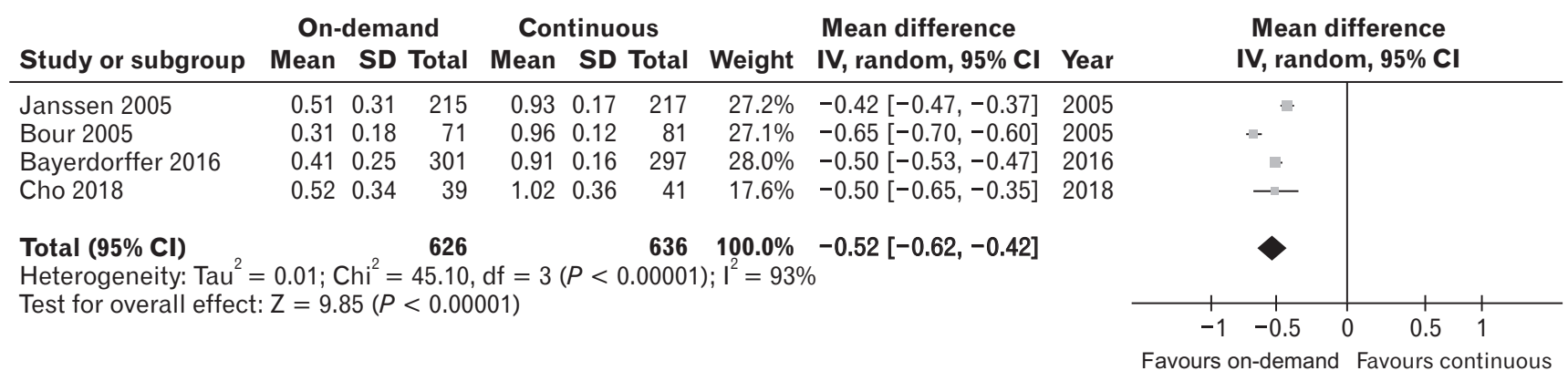

Figure 5. Forest plot of the amount of pill usage between on-demand therapy and continuous therapy. IV, inverse variance.

groups (RR, 1.02; 95\% CI, 0.90-1.14) (Supplementary Fig. 12).

\section{Discussion}

The meta-analysis of RCTs on maintenance treatment in GERD patients found no difference in treatment failure and satisfaction between the on-demand and continuous PPI maintenance groups. In terms of symptom relief, continuous therapy was more effective than on-demand therapy. However, meta-regression analysis demonstrated that the severity of esophagitis can affect the effectiveness of maintenance therapy. In NERD and mild ERD patients, treatment failure rate was similar in both therapies, and symptom relief was slightly higher in the continuous therapy group, although the clinical significance of this difference does not appear to be large $(\mathrm{NNT}=14.3)$. In terms of treatment failure and symptom relief, continuous maintenance therapy was more effective in studies including severe ERD patients. However, in Asian patients where severe ERD patients are rare, there was no difference in the effect of symptom relief between both maintenance therapies. The amount of pill usage was significantly lower in the on-demand group. Therefore, in GERD patients with NERD and mild ERD patients or Asian patients, on-demand therapy may be preferentially recommended as an effective maintenance therapy.

Although many guidelines recommend on-demand therapy as a maintenance therapy for GERD, few meta-analysis articles have reported its effectiveness. Boghossian ${ }^{10}$ reviewed the efficacy of ondemand PPI therapy versus continuous PPI therapy in NERD or mild ERD (LA grade A or B). The authors concluded that on-demand PPI therapy may increase risk of "lack of symptom control" compared with continuous PPI use (RR, 1.71; 95\% CI, 1.31-2.21), thereby favoring continuous PPI use. Khan et $\mathrm{al}^{11}$ also performed a meta-analysis comparing on-demand therapy with continuous PPI use in patients with NERD or mild ERD. Their conclusion that on-demand PPI was superior to continuous PPI
(OR, $0.50 ; 95 \% \mathrm{CI}, 0.35-0.72)$ in terms of 'discontinuation of maintenance treatment' is contrary to the results of the former study. In our study, when limited to NERD and mild ERD patients as in the 2 studies above, continuous maintenance treatment was slightly more effective in symptom relief (RR, 1.09; 95\% CI, 1.01-1.18), as in the study of Boghossian, and there was no difference between the 2 groups in treatment failure (RR, 0.85; 95\% CI, 0.43-1.66). This result was different from that of Khan's study. ${ }^{11}$ This is because van der Velden's study ${ }^{24}$ was added to our study due to the difference in inclusion criteria, and the results of this study have an influence on the outcome. However, in studies including moderate to severe esophagitis, continuous therapy showed superior efficacies in terms of treatment failure (RR, 4.24; 95\% CI, 2.32-7.70) and symptom relief (RR, 1.37; 95\% CI, 1.23-1.52). The American Gastroenterological Association guideline also reports that the use of long-term PPI has a greater benefit than harm in GERD patients with severe esophagitis or complications such as strictures. ${ }^{25}$

In subgroup analysis, 3 studies included all grades of esophagitis. However, grade $\mathrm{C}$ and $\mathrm{D}$ esophagitis patients accounted for only $6.0 \%$ (7/117) in the Nagahara's study ${ }^{20}$ and $2.5 \%$ (2/80) in Cho's study. ${ }^{16}$ In the Sjöstedt's study, ${ }^{21}$ patients with grade $\mathrm{C}$ and grade D esophagitis accounted for $17.0 \%$ (80/470) and 5.0\% (24/470) of all patients, respectively. Therefore, to confirm the effect of maintenance therapy in severe esophagitis such as LA grade C and D, the results of Sjöstedt's study ${ }^{21}$ should be referenced. Treatment failure was significantly higher (RR, 4.24; 95\% CI, 2.32-7.77), and symptom relief was significantly lower (RR, 1.37; 95\% CI, 1.23-1.52) in the on-demand PPI group compared to the continuous therapy group. Furthermore, the authors also investigated the cumulative healing rate of esophagitis for up to 6 months. The cumulative proportion of patients in remission on the continuous PPI group was $81.0 \%$ compared with only $58.0 \%$ in the on-demand PPI group ( $P$ $<0.01)$. In patients with grade $\mathrm{C}$ and $\mathrm{D}$ esophagitis, relapse rates during on-demand maintenance treatment were as high as $49.0 \%$ 
and $57.0 \%$, respectively. Therefore, in the maintenance therapy of overall GERD patients, on-demand therapy showed a similar effect to continuous therapy. However, when limited to the patients with severe esophagitis of LA grade C or D, the continuous maintenance therapy could be a better option compared with the on-demand PPI therapy.

Among the 11 studies included in the analysis, 2 were conducted in Asia. Nagahara's study ${ }^{20}$ was conducted in Japan and Cho's study ${ }^{16}$ was conducted in Korea. Both studies included all grades of erosive esophagitis, but the proportion of patients with grade $\mathrm{C}$ and D esophagitis were only $6.0 \%$ in Nagahara's study ${ }^{20}$ and $2.5 \%$ in Cho's study. ${ }^{16}$ According to the results of epidemiologic studies, the proportion of severe erosive esophagitis in Asia is very low compared to that in Western countries. From a multicenter prospective study from Korea, out of 354 erosive esophagitis patients, only 12 patients were grade $\mathrm{C}$ and 4 were grade $\mathrm{D}$, and the proportion of grade $\mathrm{C}$ and $\mathrm{D}$ among all erosive esophagitis was $4.5 \%$. ${ }^{26}$ This figure is similar to the proportion of grade $\mathrm{C}$ and $\mathrm{D}$ patients in the 2 Asian studies included in the analysis, and much lower than the proportion $(22.0 \%)$ found in the Western study by Sjöstedt et al. ${ }^{21}$ The effect of maintenance therapy in the Asian studies also shows different results from Western studies. When symptom relief was an outcome, continuous maintenance therapy was more effective than on-demand therapy in the entire study. However, the results of 2 studies from Asia showed no difference in symptom relief between the on-demand and continuous groups. Therefore, on-demand PPI therapy can be an effective maintenance treatment option in Asian patients with GERD.

Recent studies suggest that long-term PPI use may be associated with adverse events or complications, including enteric infection, bone fracture, kidney diseases, and micronutrient deficiency. ${ }^{8}$ A meta-analysis of 36 case-control studies and 14 cohort studies showed that $C$. difficile infection is associated with PPI use (OR, 1.26; 95\% CI, 1.12-1.39). ${ }^{27}$ An RCT that evaluated the long-term safety of PPIs over 2 years revealed increased events of $C$. difficile infection in PPI group (9 cases in 8791) compared to placebo group (4 cases in 8807), which did not reach statistical significance due to very few number of cases in either group (OR, 2.26, 95\% CI, 0.70-7.34). ${ }^{28}$ According to available evidences, the Food and Drug Administration and Prevention announced that $C$. difficile diarrhea may be associated with PPI use. Other adverse events such as kidney diseases, community-acquired pneumonia, and bone fractures have been demonstrated in case-control studies or cohort studies. ${ }^{29-31}$ Therefore, many GERD guidelines recommend that PPIs should be administered at the lowest effective dose. In this study, the amount of PPI used in the on-demand group was almost half that of the continuous group $(-0.52[-0.62--0.42]$ pill use/ day). Therefore, considering the various adverse effects associated with the use of long-term PPI, on-demand maintenance treatment is considered a safer and more cost-effective treatment modality than continuous maintenance treatment.

This study has several limitations. First, as described in the result section and Supplementary Table 1, 10 of the 11 studies included in analysis were open-label studies. On-demand therapy is expected to be difficult to conduct with a double-blind study. Only 1 study performed a double-blind study that showed that symptom control and quality of life were similar in the on-demand and continuous therapies. Since the open-label study is very similar to the actual clinical situation, the interpretation of the results is unlikely to be different significantly. Second, as a result of meta-analysis, the heterogeneity among the studies was high. The inclusion of patients with varying degrees of symptoms and esophagitis may have an effect on the heterogeneity as shown in the meta-regression analysis. Also, as a result of the subgroup analysis by the esophagitis grade, the heterogeneity within each group was significantly reduced. The diversity of outcome measurement also contribute to the high heterogeneity of this study. Third, no studies reported CYP2C19 polymorphism and their effect on PPI maintenance therapy. However, 2 studies used rabeprazole, which is not affected by CYP2C19 polymorphism, reported no difference between the maintenance therapies in terms of "treatment failure" and "symptom relief." Forth, only 2 Asian studies were included in this analysis, and the results between Asian and Western studies show slightly different trends as shown in the analysis. It may be difficult to apply the results of this study to Asian patients. In this regard, more research is needed in the Asian region. Finally, intermittent therapy is another recommended maintenance therapy in some guidelines, and is also known to be an effective maintenance option. However, because of lack of RCTs on the intermittent therapy, its effect cannot be compared with the on-demand or continuous therapy.

In conclusion, the on-demand PPI therapy showed similar efficacies to continuous therapy in the outcome of treatment failure, symptom relief, and patient satisfaction in GERD patients, especially in NERD and mild ERD patients, and Asian patients. The severity of esophagitis significantly influenced the effect of the 2 maintenance therapies. In particular, continuous maintenance treatment seems to be more effective in patients with severe ERD. However, on-demand therapy has the advantage of reducing the amount of PPI usage by half compared to the continuous therapy. Therefore, the on-demand PPI therapy is recommended as an 
effective maintenance treatment modality in GERD patients unaccompanied by severe esophagitis.

\section{Supplementary Materials}

Note: To access the supplementary tables and figures mentioned in this article, visit the online version of Journal of Neurogastroenterology and Motility at http://www.jnmjournal.org/, and at https://doi.org/10.5056/jnm21095.

Financial support: This research was supported by a grant of the Korea Health Technology R\&D Project through the National Evidence-based Healthcare Collaborating Agency (NECA), funded by the Ministry of Health \& Welfare, Republic of Korea (Grant No. HI19C0481, HC19C0060).

\section{Conflicts of interest: None.}

Author contributions: Conceptualization: Hye-Kyung Jung and Kwang Jae Lee; data curation: Seung Joo Kang and Chung Hyun Tae; formal analysis: Seung Joo Kang, Chung Hyun Tae, and Seung Young Kim; fund acquisition: Hye-Kyung Jung and Kwang Jae Lee; methodology: Hye-Kyung Jung; supervision: Kwang Jae Lee; writing-original draft: Seung Joo Kang; and writing-review and editing: Chung Hyun Tae and Seung Young Kim.

\section{References}

1. Locke GR 3rd. Natural history of nonerosive reflux disease. Is all gastroesophageal reflux disease the same? What is the evidence? Gastroenterol Clin North Am 2002;31(4 suppl):S59-S66.

2. El-Serag HB, Sweet S, Winchester CC, Dent J. Update on the epidemiology of gastro-oesophageal reflux disease: a systematic review. Gut 2014;63:871-880.

3. Zagari RM, Fuccio L, Wallander MA, et al. Gastro-oesophageal reflux symptoms, oesophagitis and barrett's oesophagus in the general population: the Loiano-Monghidoro study. Gut 2008;57:1354-1359.

4. Vakil N, van Zanten SV, Kahrilas P, Dent J, Jones R. The Montreal definition and classification of gastroesophageal reflux disease: a global evidence-based consensus. Am J Gastroenterol 2006;101:1900-1920.

5. Katz PO, Gerson LB, Vela MF. Guidelines for the diagnosis and management of gastroesophageal reflux disease. Am J Gastroenterol 2013;108:308-328.

6. van Pinxteren B, Numans ME, Bonis PA, Lau J. Short-term treatment with proton pump inhibitors, H2-receptor antagonists and prokinetics for gastro-oesophageal reflux disease-like symptoms and endoscopy negative reflux disease. Cochrane Database Syst Rev 2006:CD002095.

7. Dean BB, Gano AD Jr, Knight K, Ofman JJ, Fass R. Effectiveness of proton pump inhibitors in nonerosive reflux disease. Clin Gastroenterol
Hepatol 2004;2:656-664.

8. Maret-Ouda J, Markar SR, Lagergren J. Gastroesophageal reflux disease: a review. JAMA 2020;324:2536-2547.

9. Shaheen NJ, Hansen RA, Morgan DR, et al. The burden of gastrointestinal and liver diseases, 2006. Am J Gastroenterol 2006;101:2128-2138.

10. Boghossian TA, Rashid FJ, Thompson W, et al. Deprescribing versus continuation of chronic proton pump inhibitor use in adults. Cochrane Database Syst Rev 2017;3:CD011969.

11. Khan Z, Alastal Y, Khan MA, et al. On-demand therapy with proton pump inhibitors for maintenance treatment of nonerosive reflux disease or mild erosive esophagitis: a systematic review and meta-analysis. Gastroenterol Res Pract 2018;2018:6417526.

12. Liberati A, Altman DG, Tetzlaff J, et al. The PRISMA statement for reporting systematic reviews and meta-analyses of studies that evaluate health care interventions: explanation and elaboration. Ann Intern Med 2009;151:W65-W94.

13. Higgins JP, Altman DG, Gøtzsche PC, et al. The cochrane collaboration's tool for assessing risk of bias in randomised trials. BMJ 2011;343:d5928.

14. Bayerdörffer E, Bigard MA, Weiss W, et al. Randomized, multicenter study: on-demand versus continuous maintenance treatment with esomeprazole in patients with non-erosive gastroesophageal reflux disease. BMC Gastroenterol 2016;16:48.

15. Bour B, Staub JL, Chousterman M, et al. Long-term treatment of gastro-oesophageal reflux disease patients with frequent symptomatic relapses using rabeprazole: on-demand treatment compared with continuous treatment. Aliment Pharmacol Ther 2005;21:805-812.

16. Cho JH, Koo JY, Kim KO, Lee SH, Jang BI, Kim TN. On-demand versus half-dose continuous therapy with esomeprazole for maintenance treatment of gastroesophageal reflux disease: a randomized comparative study. Medicine (Baltimore) 2018;97:e12732.

17. Hansen AN, Bergheim R, Fagertun H, Lund H, Wiklund I, Moum B. Long-term management of patients with symptoms of gastrooesophageal reflux disease -- a Norwegian randomised prospective study comparing the effects of esomeprazole and ranitidine treatment strategies on health-related quality of life in a general practitioners setting. Int J Clin Pract 2006;60:15-22.

18. Janssen W, Meier E, Gatz G, Pfaffenberger B. Effects of pantoprazole $20 \mathrm{mg}$ in mildgastroesophageal reflux disease: once-daily treatment in the acute phase, and comparison of on-demand versus continuous treatment in the long term. Curr Ther Res Clin Exp 2005;66:345-363.

19. Morgan DG, O’Mahony MF, O’Mahony WF, et al. Maintenance treatment of gastroesophageal reflux disease: an evaluation of continuous and on-demand therapy with rabeprazole $20 \mathrm{mg}$. Can J Gastroenterol 2007;21:820-826.

20. Nagahara A, Hojo M, Asaoka D, Sasaki H, Watanabe S. A randomized prospective study comparing the efficacy of on-demand therapy versus continuous therapy for 6 months for long-term maintenance with omeprazole $20 \mathrm{mg}$ in patients with gastroesophageal reflux disease in Japan. Scand J Gastroenterol 2014;49:409-417.

21. Sjöstedt S, Befrits R, Sylvan A, et al. Daily treatment with esomeprazole is superior to that taken on-demand for maintenance of healed erosive 
oesophagitis. Aliment Pharmacol Ther 2005;22:183-191.

22. Szucs T, Thalmann C, Michetti P, Beglinger C. Cost analysis of longterm treatment of patients with symptomatic gastroesophageal reflux disease (GERD) with esomeprazole on-demand treatment or esomeprazole continuous treatment: an open, randomized, multicenter study in Switzerland. Value Health 2009;12:273-281.

23. Tsai HH, Chapman R, Shepherd A, et al. Esomeprazole $20 \mathrm{mg}$ on-demand is more acceptable to patients than continuous lansoprazole $15 \mathrm{mg}$ in the long-term maintenance of endoscopy-negative gastro-oesophageal reflux patients: the COMMAND study. Aliment Pharmacol Ther 2004;20:657-665.

24. van der Velden AW, de Wit NJ, Quartero AO, Grobbee DE, Numans ME. Pharmacological dependency in chronic treatment of gastroesophageal reflux disease: a randomized controlled clinical trial. Digestion 2010;81:43-52.

25. Freedberg DE, Kim LS, Yang YX. The risks and benefits of long-term use of proton pump inhibitors: expert review and best practice advice from the American gastroenterological association. Gastroenterology 2017;152:706-715.
26. Seo GS, Jeon BJ, Chung JS, et al. The prevalence of erosive esophagitis is not significantly increased in a healthy Korean population - could it be explained?: a multi-center prospective study. J Neurogastroenterol Motil 2013;19:70-77.

27. Cao F, Chen CX, Wang M, et al. Updated meta-analysis of controlled observational studies: proton-pump inhibitors and risk of Clostridium difficile infection. J Hosp Infect 2018;98:4-13.

28. Moayyedi P, Eikelboom JW, Bosch J, et al. Safety of proton pump inhibitors based on a large, multi-year, randomized trial of patients receiving rivaroxaban or aspirin. Gastroenterology 2019;157:682-691, e2.

29. Hart E, Dunn TE, Feuerstein S, Jacobs DM. Proton pump inhibitors and risk of acute and chronic kidney disease: a retrospective cohort study. Pharmacotherapy 2019;39:443-453.

30. Nguyen PA, Islam M, Galvin CJ, et al. Meta-analysis of proton pump inhibitors induced risk of community-acquired pneumonia. Int J Qual Health Care 2020;32:292-299.

31. Brusselaers N, Lagergren J, Engstrand L. Duration of use of proton pump inhibitors and the risk of gastric and oesophageal cancer. Cancer Epidemiol 2019;62:101585. 